\title{
Mitigating the Hospital Area Communication's Interference using Cognitive Radio Networks
}

\author{
Dramane Ouattara*, Minh Thao Quach*, Francine Krief*, Mohamed Aymen Chalouf ${ }^{\ddagger}$ and Hicham Khalife ${ }^{\dagger}$ \\ * University of Bordeaux, France, Email: dramane.ouattara@1abri.fr, minh-thao.quach@labri.fr, francine.krief@labri.fr. \\ $\ddagger$ University of Rennes 1, France, Email: mohamed-aymen.chalouf@irisa.fr. \\ †Thales France, Email: hicham.khalife@thalesgroup.com.
}

\begin{abstract}
The communication infrastructures and devices in hospitals are becoming increasingly wireless because of doctors and nurses are mobile in their work. On the other hand, the hospital patient's communications or their monitoring generates lot of electromagnetic waves. In this context, deployment of wireless networks should not only meet some performance requirements but should also be aware and able to control the interferences. Cognitive Radio Networks (CRNs) seem to be suitable for delivering high-quality communication services and mitigating radio interferences in hospital environment. In this paper, we discuss the design of hospital networks above CRNs to overcome current constraints.
\end{abstract}

\section{INTRODUCTION}

The growing interest for a radical shift from wired to wireless medical devices is promising a care service improvement and more mobility for patients and staffs. Thus, wireless systems become increasingly intrinsic part of modern medical devices such as insulin pumps, cardiac defibrillators or pacemakers. However, their communications could greatly increase electromagnetic interference with other critical medical equipments. There is therefore a need to mitigate potential risks of electromagnetic interference between the patients wireless devices and sensitive medical equipment in clinical health care environment. New communication policies should be developed to ensure efficient communications while minimizing electromagnetic interference. The Cognitive Radio Networks [1] (CRNs) have recently become the forefront of wireless research as it is expected to reduce connection cost while offering new applications, higher throughput and capabilities for avoiding interference. In most common configurations, a Cognitive Radio user (CR) exploits the spectrum bands transparently to legacy users, called Primary Users (PU). Using its flexibility, CR explores different technologies and standard to identify free frequency bands for opportunistically ensuring its transmissions while avoiding interference with the other users. Our proposal is to deploy this technology in a hospital environment with sensitive medical devices as primary users (PUs) and the patients mobile equipments as the CRs. Thus, the medical devices vulnerability areas should be clearly, the overlap regions in the $\mathrm{CR}$ mobile nodes coverage areas. Accordingly, we firstly compute the overlap region to characterize these areas. Secondly, we estimate the positions of the patients CR mobile equipments through the "Grey Model" theory. Finally, knowing the positions of the medical equipments and CR mobile emitters, our proposed framework allows decision-making to mitigate radio interferences. The remainder of this paper is structured as follows: Section II gives an overview on the related work. We illustrate the CRNs deployment in a hospital context in Section III, then we describe our proposed interference management plan in Section IV. Functional analysis and simulation results are discussed in Section V. Section VI concludes and lists future work.

\section{RELATED WORK}

Several studies have given guidelines for the use of cognitive radio in healthcare environment for interference mitigation. A health care automation networks architecture and design has been introduced in [2]. To overcome the cognitive radio overlapping interferences, this work proposes to model the spectrum sharing between the devices as a graph colouring problem. Thus, two adjacent transmitter nodes or devices cannot share the same frequency band due to mutual interference risks. This work has an interesting theoretical approach but the graph colouring is a NP-complete problem [3] and all algorithms for solving NP-complete problems have exponential processing time and are difficult to solve in practice. An architecture of a wireless communication link between the ambulance and the hospital based on CRNs was studied in [4]. This article shows the importance of having direct broadband communication link between the ambulance and the hospital in case of brain stroke disturbance for blood pressure, glucose level and video image transmissions. The cognitive radio module in this case, will allow the suitable empty frequency band after the spectrum sensing on the available channels. The paper highlights the CRNs performance in patients monitoring context and the unavoidable electromagnetic interference remains less concerned. Similar work was done in [5] where the proposed solution addresses the performance with the permanent connectivity issue. The proposal presented in [6] demonstrates the ability of cognitive radio to improve Wireless Body Area Networks (WBAN) performances. An example of e-health wireless communication system, based on cognitive radio, deployed in a hospital environment is described in [7]. The authors suggest a communication protocol based on centralized architecture where cognitive radio node server manages the PUs and CRs transmissions with queuing packets mechanism. This approach offers more practical method to overcome the hospital area interferences but may soon face to the scalability problem. Autonomous interference management considering the patients mobility has not been broadly investigated. We propose an interference-aware management framework which is able to make decision on selecting frequency bands and adapting transmission parameters of CR devices in order to reduce the patient's communication interference on sensitive medical devices. 


\section{THE PROPOSED FRAMEWORK}

Figure 1@ shows the interference caused by the patient's communications on the medical equipments. In fact, medical devices installed around the patient are exposed to electromagnetic disturbances during the transmissions of the patient devices. Figure 1 b describes the proposed framework for mitigating this interference using cognitive radio for dynamic adaptation of the transmission parameters (e.g. power). More precisely, we propose the use by patients of mobile equipments with cognitive radio capabilities. The patient communications could be classified into two categories, namely the patient's personal communications and the patient's vital signs monitoring data transmissions through the Wireless Personal Area Networks (WPAN). The CR mobile node could serves as the WPAN relay node to the outside networks or as the patient's personal communication-tool. Figure 1 b illustrates our planned CRNs deployment mechanism in a hospital environment where $\mathrm{CR}$ devices are used to perform the patient's communications. The Planned Transmitters (PT) serve as cognitive radio access points to the patient's CR mobile nodes (CM). The access networks (e.g. WiFi, GSM, ZigBee) cover the hospital area for non-cognitive radio devices and medical equipments usage. Our planned CRNs opportunistically exploits these underlying multiple technologies (access networks) while avoiding the interferences. A cognitive radio equipment is able to periodically scan and measure through its sensing module the interference level on a given frequency band, to then adjust its transmission parameters accordingly. The Planned Transmitters maintain a database that describes the internal and external radio environment characteristics. As instance, for a given frequency band and for each zone, taking into account the type of installed medical devices is associated an acceptable interference limit $I_{L}$. The interference on this frequency band includes the sum of all noises caused by the different signals. Thus, the interference caused by the patients fitted cognitive radio communication nodes through its transmissions is noted $I_{t r}$ and can be expressed by[8]:

$$
I_{t r}\left(f_{c}, W\right)=\frac{P_{m}\left(f_{c}, W\right)}{K W}
$$

where $P_{m}\left(f_{c}, W\right)$ is the average transmission power, for bandwidth $\mathrm{W}$ at center frequency $f_{c}$, and $\mathrm{K}$ is the Boltzmann's constant in Joules $\left(K=1.38 \times 10^{-23}\right)$.

Each planned CRNs cell, with fixed access point as described on figure 1 (b), is modelled to manage the interference level as follows:

$$
I_{t r}\left(f_{i}, W_{i}\right)+\frac{M_{i} P_{m}}{K W_{i}} \leq I_{L}\left(f_{i}\right)
$$

for $i \in\{1, \ldots, n\}$ transmitters, $M_{i}=[0,1]$ denotes the attenuation factor due to the fading and path loss between the $\mathrm{CR}$ mobile transmitter and the medical receiver.

\section{THE PROPOSED INTERFERENCES MANAGEMENT PLAN}

Figure 2 summarizes the management plan which is based on a set of modules and implemented on each Planned Transmitter (PT). Measurement function controls and defines the parameters (e.g. transmission power, center frequency, bandwidth, modulation techniques) and calculates the distance or the location of the CR mobile node (CM), the overlap region and evaluates the Interference Level $\left(I_{L}\right)$ as defined in

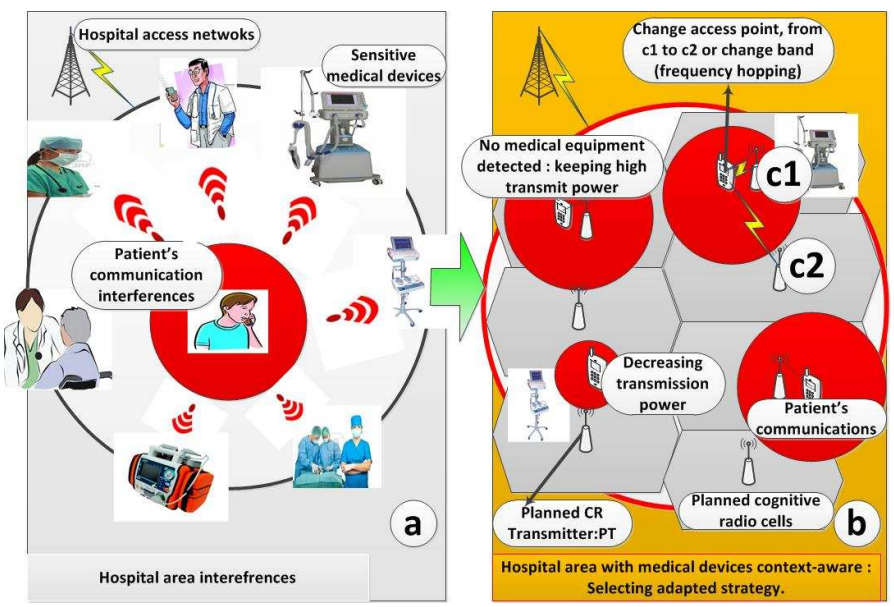

Fig. 1: CR deployed in hospital context

equation (2). Once measured, theses metrics are stored in the database and they are periodically updated. The distance and the location metrics are more explained in section V. The other parameters are more easily programmable and measurable by the cognitive radio equipment. According to these varying parameters, the strategy function defines strategies to be adopted for ensuring high quality of the transmission at a patient's mobile node, while reducing interferences. A list of strategies related to the radio environment constraints and a scheduling process based on the applications priority can be established. The channel or the central frequency change, the transmit power adjustment (increase or decrease), the any transmitting signal stopping on any frequency band are the obvious actions examples, that validate the strategies. A strategy can be easily expressed by the rules of logic programming. Thus, a strategy is composed of an action and a set of conditions to be satisfied. Actions are operations to perform in order to change the state of CR node parameters. To each Action, we associate a set of Preconditions (REQUIRE) and Postconditions (ENSURE). Preconditions set rules or conditions that must be satisfied. The Postconditions describe the modified state of the strategy. Two examples of strategies leading to the execution of two different actions, decreasing the transmission power (Algorithm 1) and changing the transmission band (Algorithm 2) are given below:

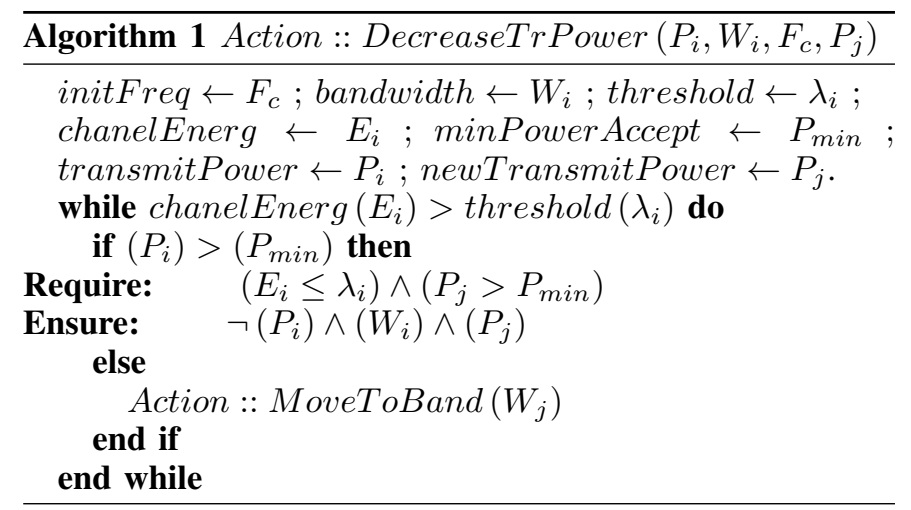

Algorithm 1 describes the strategy which leads to an adaptation (decrease) of the transmission power to meet the requirements of the interference limit (threshold), compare to the detected energy on the channel (chanelEnerg). 

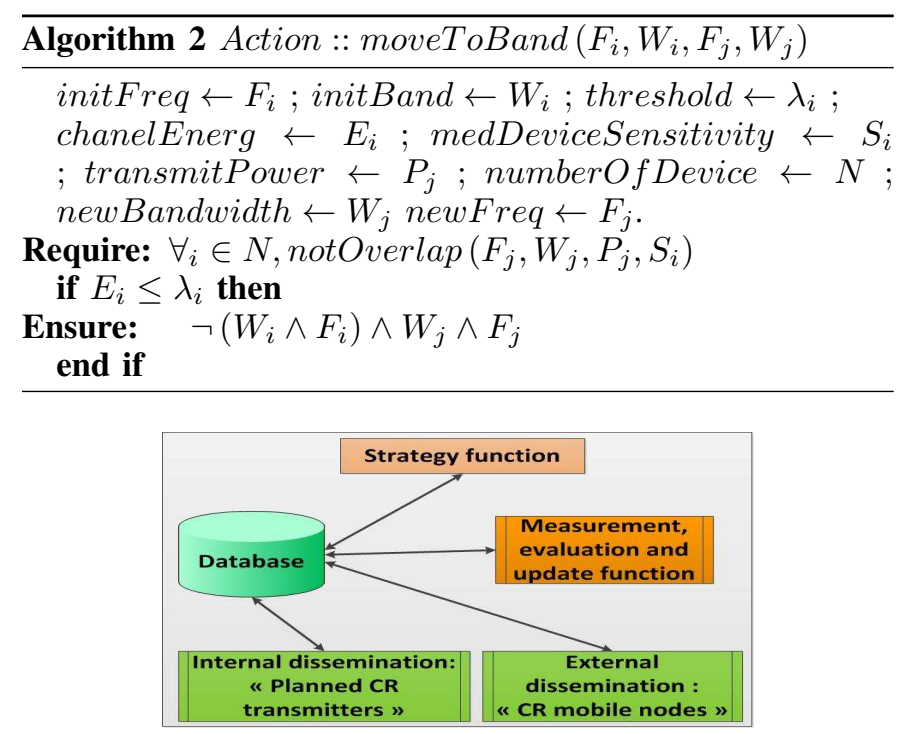

Fig. 2: Interference management plan

Algorithm 2 shows the frequency hopping when the minimum transmission power (minPowerAccept) required for ensuring better transmissions is no longer guaranteed. Also, the function notOverlap ensures that the new frequency band chosen complies with the overlap constraints and therefore generates no interference to medical equipments (medDeviceSensitivity) in this area

The internal dissemination function shares updated information and strategies stored in the database between CR transmitters. The $\mathrm{CR}$ mobile nodes positions, theirs transmission powers, a new medical device installation, could be the data in exchanged messages. From the information exchanged and the measurements realised, the CR transmitter recalculates locally its parameters and adapts its strategies. The dissemination function is executed only when a new event is observed such as the arrival of a CR mobile node for reducing information exchanged rate. The external dissemination concerns the information exchanged between the CR transmitters (PT) and the patient's CR mobile nodes. The information shared may contain among others, the supported interference limit on the available bands and the maximum permitted transmission power on each cell. Therefore, the cognitive radio signalling protocols are used for connection initiation, for synchronisation and also for the interference management plan information exchanges.

\section{FUnCTIONAL ANALYSIS AND RESULTS}

As shown in the proposed framework, the hospital area interference management is based on a set of functions whose most important are detailed in this section.

\section{A. The overlap regions characterization}

The aim of accurately deriving the overlap coverage areas of CR transmitters and patient's mobile node transmissions is to characterize the potential interference on sensitive medical devices. We call an overlap region the intersection of the two disks created by the coverage area of a CR mobile node and the planned CRs transmitters. The obtained surface defines the region where medical devices or medical receivers seem more exposed to interference.

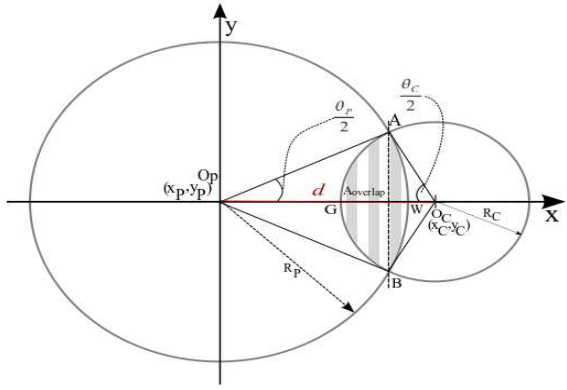

Fig. 3: General overlap case

1) Problem statement and formulation: We consider a single CR mobile node and a single planned CR transmitter knowing that the treatment is distributed and results of locally computation performed at each planned CR transmitter will be shared for the overall network better coordination. We also assume that the coverage area of the mobile node and the CR transmitters forms a perfect circle whose center is located at the position of the emitter.

We denote by $O_{P}$ of co-ordinates $\left(x_{P}, y_{P}\right)$ and $O_{C}$ with $\left(x_{C}, y_{C}\right)$ the position of the planned transmitter and mobile $\mathrm{CR}$ patient's node $(\mathrm{CM})$ respectively, $d$ the distance between these two points, RP and RC the radius of their coverage disk respectively. Our goal is to calculate the overlap region and observe the impact of the patient's CR mobile node transmissions on the sensitive hospital receivers within the area. The used notations are summarized below:

\begin{tabular}{l|l} 
Symbols & Descriptions \\
\hline \hline$O_{P}\left(x_{P}, y_{P}\right)$ & $\begin{array}{l}\text { Center and corresponding co-ordination of a planned } \\
\text { transmitter PT }\end{array}$ \\
\hline$O_{C}\left(x_{C}, y_{C}\right)$ & $\begin{array}{l}\text { Center and corresponding co-ordination of a patient's } \\
\text { cognitive mobile node CM }\end{array}$ \\
\hline$R_{P}$ & Radius of the PT power transmission \\
\hline$R_{C}$ & Radius of the CM power transmission \\
\hline$d$ & Distance between the center of a PT and a CM \\
\hline$\theta_{P}$ & Angle of the sector created by the intersection of PT \\
\hline$\theta_{C}$ & Angle of the sector created by the intersection of CM \\
$B\left(x_{A}, y_{A}\right)$ & Intersection points of CM and PT \\
\hline$G\left(x_{G}, y_{G}\right)$ & Inner point and on the boundary of the overlapped region \\
\hline$W\left(x_{W}, y_{W}\right)$ & Outer point and on the boundary of the overlapped region \\
\hline
\end{tabular}

2) The overlapping region computation base: In this computations we consider that the PT and CM positions are known, the radii are fixed and the PT transmitter is located at the origin of the coordinate system. The general case highlighted in Figure 3, is obtained when the following condition is satisfied:

$$
d<R_{P}+R_{C} \text { and } d>R_{P} \text { and } d>R_{C}
$$

Using classical geometry, we calculate the general overlap region between PT and CM in equation (4).

$$
\begin{aligned}
A_{\text {Overlap }}= & \frac{\theta_{C}}{2} R_{C}^{2}-R_{C}^{2}|\cos \beta| \cos \frac{\theta_{C}}{2}+ \\
& \frac{\theta_{P}}{2} R_{P}^{2}-R_{C}|\cos \beta| R_{P} \cos \frac{\theta_{P}}{2}
\end{aligned}
$$

Where $\theta_{C}$ and $\theta_{P}$ are the angles formed at $O_{C}$ and $O_{P}$ with points $A$ and $B$ respectively, whereas $\alpha$ and $\beta$ are intermediate 
variables for our computation given below:

$$
\begin{array}{lrl}
\sin \alpha=\frac{x_{C}}{\sqrt{x_{C}^{2}+y_{C}^{2}}} ; & \sin \beta=\frac{R_{P}^{2}-\left(R_{C}^{2}+x_{C}^{2}+y_{C}^{2}\right)}{2 * R_{C} * \sqrt{x_{C}^{2}+y_{C}^{2}}} \\
\sin \frac{\theta_{C}}{2}=\frac{R_{C}}{R_{P}} *|\cos \beta| ; & \sin \frac{\theta_{P}}{2}=|\cos \beta|
\end{array}
$$

3) The overlapping size and it's impact evaluation: The relationship between the overlap phenomena and its impact to the medical devices is investigated using OMNeT++ simulator with the integrated MiXim framework [9]. The simulation was deployed in a playground size $3000 m \times 3000 m$, the PT transmits on a typical $802.11 \mathrm{~b}$ bands with the maximum transmission power of $110.11 \mathrm{~mW}$ that corresponds to a coverage range of $475.8 \mathrm{~m}$. We developed a CM node on top of an adaptive 802.11 multi-channel implementation that allows nodes to switch between channels under specific conditions. Note here that the IEEE 802.11 was selected for simplicity reasons; However, our results can be generalized over any spectrum band. Cognitive radio transmission power was fixed at $55.11 \mathrm{~mW}$ that covers a distance of $377.77 \mathrm{~m}$. This coverage distance is computed according to the maximum transmission power, wave length, the path lost coefficient and a threshold for the minimal receiving power. The built-in path loss propagation was used with default path loss coefficient alpha of 3.5. All the physical values were also kept by default as pre-defined in MiXim while the transmission power of the CM node was modified and set to be lower than the PTs transmission power. We placed the medical device receivers in the disk area around the PT transmitter following various distributions.

4) Simulation results: Figure 4 (a) shows results for a scenario where over a single channel network we modify the overlap size and study its impact on medical device receivers. We consider Uniform and Poisson distributions to deploy hospital receiver nodes around the PT radio emitter. The ratio of impacted medical receivers by the CMs transmissions grows linearly from $30 \%$ to more than $90 \%$ when the overlap size increases as observed in Figure 4 (a). Figure 4 (b) corresponds to the scenario where two channels are available. These result shows that the multichannel exploration with cognitive radio could help reducing interference. In fact, unlike to the single channel scenario, the ratio of impacted medical devices varies from almost $34 \%$ to almost $40 \%$ on channel1 and from $10 \%$ to almost $15 \%$ on channel2. Also, we observe that the ratio of affected medical devices is almost independent of the number of medical receivers and is not related to the distribution type. These important observations reinforce our principle that consists in dynamic transmission power adaptation according to the interference limit and CM radio position.

\section{B. The patient's $C R$ mobile nodes position calculating}

Computing the position of the CM nodes remains a challenge because the existent geolocation tools are inappropriate. In fact, based on the literature [10], for calculating the distance or users position coordinates, it has been assumed that the average signal strength is a stationary Gaussian process. Therefore, based on the relationship between transmission power and reception power that is approximated in free space by [11]: $P_{r}(d)=\frac{P_{t} G_{t} G_{r} \lambda^{2}}{(4 \pi d)^{2} L}$, where $P_{r}$ and $P_{t}$ are respectively received and transmitted powers, $G_{r}$ and $G_{t}$ denote the antenna gains of receiver and transmitter, $L$ the system loss factor (filter losses, antenna losses, path-loss), $\lambda$ is the wavelength of the transmit signal in meter and $d$ the distance between the transmitter and the receiver is in this case easily deducted. But with cognitive radio, an inevitable non-stationary signal strength is required with self-adaptation of transmission power. Considering the uncertainty induced by the environment and the unstable transmission-parameters context, we opted to modelling this environment with the Grey Model for distance $d$ estimation and prediction.

1) The Grey Model: The Grey Model theory [12] is known for the analysis of problems with incomplete or uncertain information and the $\operatorname{GM}(1,1)$ is the most used in prediction systems.

2) The problem modelling with the $\operatorname{GM}(1,1)$ :

- The system takes as input a sequence of values, the received power that characterizes the received CM's signal; with $X^{0}=\left[x^{0}(1), x^{0}(2) S, \ldots, x^{0}(n)\right]$, the initial distribution corresponding to each $\mathrm{CM}$ received power from the overlap region and $x^{0}(d)$ their average distance.

- From the initial distribution, a new sequence $X^{1}$ is generated by the system with new values $X^{1}=$ $\left[x^{1}(1), x^{1}(2), x^{1}(3), \ldots, x^{1}(n)\right]$, which corresponds to the aggregation of the preliminary results on a given period $\mathrm{T}$ and gives a more accurate new estimation.

- The first order differential equation obtained from $X^{1}$ is given by: $\frac{d x^{1}(t)}{d t}+a x^{1}(t)=b$ where $\mathbf{a}$ is the coefficient that reflects the trend and $\mathbf{b}$ is the predictive control coefficient expressing the portion of the information known/unknown part of the model.

- The parameter estimation or coefficients of the matrix $[a, b]^{T}$ can be obtained by the method of least squares knowing that $[a, b]^{T}=\left(B^{T} B\right)^{-1} B^{T} y_{N}$, where

$$
\begin{aligned}
& B=\left[\begin{array}{cr}
-\frac{1}{2}\left(x^{1}(1)+x^{1}(2)\right. & 1 \\
-\frac{1}{2}\left(x^{1}(2)+x^{1}(3)\right. & 1 \\
\ldots \ldots . & . \\
-\frac{1}{2}\left(x^{1}(n-1)+x^{1}(n)\right. & 1
\end{array}\right] \text { and } y_{N}= \\
& {\left[x^{0}(2), x^{0}(3), x^{0}(4), \ldots, x^{0}(n)\right]^{T} ; \mathrm{n} \text { being the size of the }} \\
& \text { sequence. }
\end{aligned}
$$

- The distance $(t=d)$ estimation function becomes: $\hat{x}^{1}(t)=\left(x^{1}(1)-\frac{b}{a}\right) e^{-a t}+\frac{b}{a}$;

- The distance variation according to the received power could be obtained by: $\hat{x}^{0}(d+1)=\hat{x}^{1}(d+1)-\hat{x}^{1}(d)$.

3) Simulation results: We used GNU Octave, a free MatlabSimulink version, distributed under the terms of the GNU General Public License (GPL)[13]. The Add White Gaussian Noise (AWGN) function randomly generates the received power by adding white Gaussian noise to the vector signal. Thus, knowing the PT position and its transmitting power together with the CM transmitting power, the PT compute the overlap region with CM's positions. Figure 4 (c) shows the relationship between the received signal strength and the distance. The results of this simulation allows to estimate the different positions of the CM transmitters and to characterize the overlap region with CM's mobility. The sensitivity range for this case has a limit which approaches the $-100 \mathrm{dBm}$. These results can also be the basis for adaptive transmission power selection. 


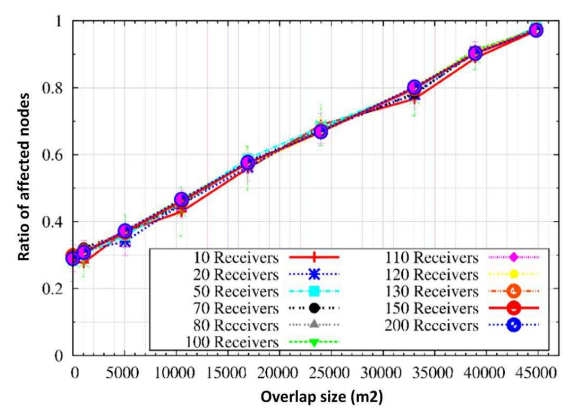

(a) Single Channel Distribution

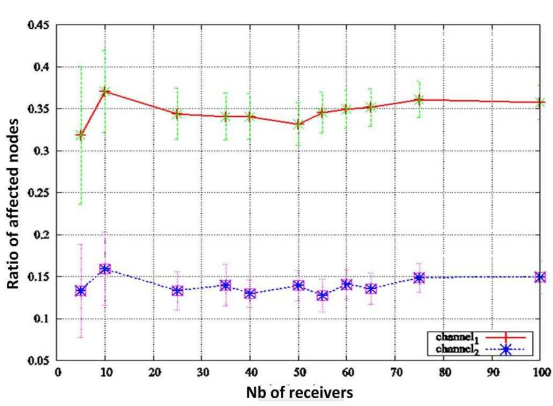

(b) Multi Channel Distribution

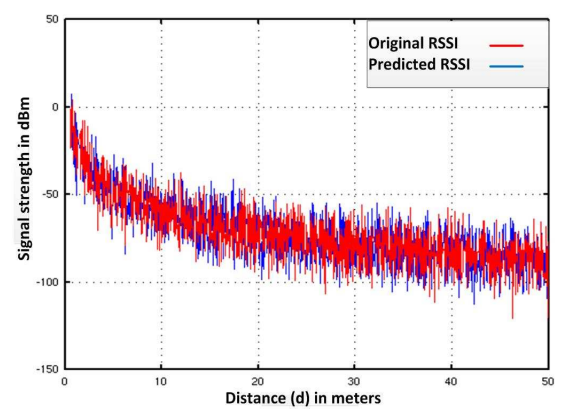

(c) Distance prediction

Fig. 4: Simulation results

\section{Strategy adoption}

The choice of strategy is based on various criteria such as the QoS, the network performance, the internal and the external radio constraints. The most interesting strategies emerging in this work are emitting power control and frequency hopping for interference avoidance. Controlling the transmission power for the CMs nodes has been significantly raised in section $\mathrm{V}(\mathrm{B})$ and the results (Figure 4-c) show that, on the basis of a given position relative to medical device receivers, $\mathrm{CM}$ node can dynamically select a suitable transmission power that considers the supported interference limit $\left(I_{L}\right)$. The frequency hopping was experimented on our cognitive radio platform.

1) Cognitive radio platform: Frequency hopping is a method of changing carrier frequencies periodically or when needed. By changing its transmission frequency band, the radio system (CM) avoids interference with the medical devices with which it shares the same channel. To implement the frequency hopping, we used a platform based on GNU Radio and Ettus universal peripherals USRP [13]. This platform is composed of five boxes USRP-1. Each USRP-1 is associated with two external omnidirectional antennas RFX2400 and RFX900, with USB port connection. Computers connected through USB ports to these devices work with gnuradio software.

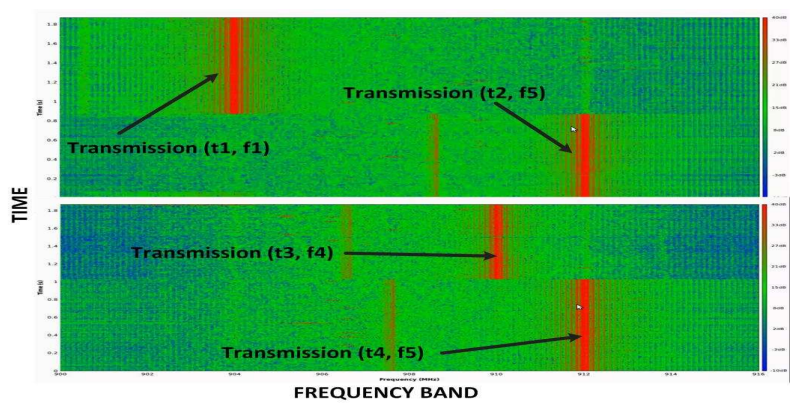

Fig. 5: Frequency hopping strategy

2) Experiment results: To test our frequency hopping program without interfering any medical device, we decided to use the $900 \mathrm{MHz}$ to $916 \mathrm{MHz}$ range. This part of the spectrum is divided into eight $2-\mathrm{MHz}$ sub-bands. Then, we start transmitting on the first band (signalling channel) and generate the seed number. The energy detection scheme defined in the cognitive radio sensing module is used. We use the AWGN signal generator to create interference in the band in which we were transmitting, and we observe the frequency hopping from the band centred on frequency $f_{1}$ at the time $t_{1}$ to that centred on $f_{5}$ at $t_{2}$ as presented at Figure 5.

\section{CONCLUSION}

In this work, we proposed a framework for interference mitigation in hospital context. We described the defined CR node functions allowing hospital environment communications without interferences. Thus, a function for calculating the overlapping area was considered. This function is powered by the patient's location coordinates given by Grey Model technique. On the basis of these results and environmental constraints, the strategy-function could adapt the transmission power or perform a frequency hopping. The obtained results provide a basis for a new hospital autonomous communication model without having interference disturbance. We are working on a new cognitive radio signalling protocol more adapted to current context and we plan to study the proposed solution cost in term of energy consumption for the CRs mobile equipments and to evaluate its throughput performance.

\section{ACKNOWLEDGMENT}

This work is supported by the LICoRNe project, funded in part by the French National Research Agency - ANR (Agence Nationale de la Recherche).

\section{REFERENCES}

[1] Ian F. Alyikdiz and Won-Yeol and Kaushik R. Chowdhury, CRAHNs: Cognitive Radio Adhoc Networks, AdhocNet 2009.

[2] Z. Dong, Shamik S. Anand, Kai H.: Cognitive Radio Mobile Ad Hoc Networks in Healthcare: chapter 13, Springer 2011.

[3] Sharma, P.C. and Chaudhari, N.S. : Phase Transition in Reduction between 3-SAT and Graph Colorability for Channel Assignment in Cellular Network : (CICN), Fourth Int. Conf., 2012.

[4] Van Moer, W. and Bjorsell, N. : Saving lives by integrating cognitive radios into ambulances : (MeMeA), 2012 IEEE Int. Symp. 2012.

[5] D. Ouattara, F. Krief, M. A. Chalouf and O. Hamdi : spectrum sensing improv. in CR Netw. for real-time patients monitoring, MobiHealth 2012.

[6] Feng Ji. and Liu Wei and Li Yang : Performance Enhancement of WBAN System Combined with Cognitive Radio : CMC '10 Int. Conference on Communications and Mobile Computing, (2010)

[7] Phunchongharn, P. and Hossain, E. and Niyato, D.: A cognitive radio system for e-health applications in a hospital environment : Wireless Communications, IEEE, vol.17 : pp.20-28,(2010)

[8] T.C. Clancy, Achievable capacity under the interference temperature model, IEEE Intern. Conf., INFOCOM 2007.

[9] ICST, Brussels, Belgium : OMNeT++ 2009: Proceedings of the 2nd International Workshop on OMNeT++ (hosted by SIMUTools 2009.

[10] Hatami, A.; Pahlavan, K.; On RSS and TOA based indoor geolocation a comparative performance evaluation, WCNC IEEE, 2006.

[11] Ekram H., Dusit N. : Dynamic spectrum access and management, Cognitive Radio Networks : Cambridge University Press, 2009.

[12] Deng Julog : The Basis of Grey Theory : Huazhong University of Science and Technology Press, 2002.

[13] http://gnuradio.org/ ; http://www.gnu.org/software/octave/about.html 